\title{
Towards a Human-centered E-Commerce Personalization Framework
}

\author{
Marios Belk
ent of Computer Science \\ Marios Belk
Department of Computer Science
University of Cyprus (UCY) \\ Marios Belk
epartment of Computer Science
University of Cyprus (UCY) \\ Nicosia, Cyprus \\ belk@cs.ucy.ac.cy
}

\author{
Panayiotis Andreou \\ Department of Computing \\ University of Central Lancashire Cyprus (UCLan Cyprus) \\ Larnaca, Cyprus \\ pgandreou@uclan.ac.uk
}

\author{
Panagiotis Germanakos \\ Suite Engineering UX, Products and Innovation \\ SAP SE, Walldorf, Germany and \\ Dept. of Computer Science \\ University of Cyprus (UCY) \\ Nicosia, Cyprus \\ panagiotis.germanakos@sap.com
}

\author{
George Samaras \\ Department of Computer Science \\ University of Cyprus (UCY) \\ Nicosia, Cyprus \\ cssamara@cs.ucy.ac.cy
}

\begin{abstract}
This paper presents a personalization framework, namely PersonaWeb that adapts the visual and interaction design of E-Commerce Web environments based on human cognitive differences. In particular, it describes a user model formalization that incorporates a set of human cognitive factors (i.e., cognitive styles and working memory capacity) and an adaptation engine that personalizes the visual and interaction design attributes of E-Commerce product views. The proposed framework has been applied in a real-life E-Commerce Web-site and two subsequent user studies were conducted in which 135 users interacted with the personalized and the original (non-personalized) version of the same Web environment. Results indicate the added value of personalizing content and functionality of E-Commerce product views in terms of users' task completion performance.
\end{abstract}

Keywords-web personalization; human cognitive differences; e-commerce; usability; user study.

\section{INTRODUCTION}

Since the late $1990 \mathrm{~s}$, personalization strategies have been embraced by researchers and practitioners aiming to improve the user experience and tackle the one-size-fits-all issues in ECommerce systems, by providing personalized products, content presentation and functionality that are bootstrapped to the needs and characteristics of each user [1, 2]. The primary factors being modeled for personalization in E-Commerce systems include, information about the users (e.g., interests, preferences, needs and goals), information about the interaction device (e.g., screen size, input type), and information about the context of use (e.g., physical, social). Yet, intrinsic human factors (e.g., cognitive processing abilities) that define users as individuals have not been widely studied and applied in ECommerce personalization, although research in other disciplines revealed a main effect of human factors on users' task performance, preference, comprehension and learning [3$6]$.

In this context, motivated by existing theories on individual differences which argue that users have different cognitive processing styles and abilities, we suggest that in the context of E-Commerce personalization, such intrinsic human factors could provide a strategic perspective for comprehensively modeling users since human-computer interactions in these settings are primarily processed on a cognitive level (users are required to process and comprehend information, and take decisions).

Accordingly, the paper presents a personalization framework, namely PersonaWeb [7], which aims at improving the shopping experience of users by adapting the visual and interaction design aspects of E-Commerce Web environments based on the users' cognitive processing characteristics. Among a high number of different theories on human cognitive differences [8], two particular cognitive factors are used as the theoretical basis for modeling the users' cognitive differences: cognitive styles and working memory capacity. Cognitive styles are based on Riding's Cognitive Style Analysis (CSA) [8, 9], which distinguishes users as Verbals that can proportionally process textual content more efficiently than images, or as Imagers that can proportionally process image-based content more efficiently than exclusively illustrated text-based content. The CSA further distinguishes users as Wholists that prefer and tend to structure incoming information as a whole to get the big picture, or as Analysts that prefer and tend to structure the incoming information in detail. Furthermore, working memory is based on Baddeley's working memory model [10] with the aim to distinguish users that have enhanced working memory capacity or limited working memory capacity.

Based on the aforementioned individual differences, PersonaWeb personalizes the visual and interaction aspects of E-Commerce product views. For example, taking into account the users' Verbal/Imager cognitive styles, can lead to a different representation of product views (e.g., with an exclusive textual representation in the case of Verbal users or a graphical illustration of content in the case of Imager users). Furthermore, by taking into account the users' working memory capacity we can increase or decrease the quantity of the presented information. The rest of the paper presents: (i) the architecture of the PersonaWeb framework including its main modules and components; (ii) the formalization of a user model that includes the users' cognitive processing factors and an 
adaptation engine that applies rules for dynamically transforming the visual and interaction design of Web-pages; and (iii) the evaluation of the PersonaWeb framework with two subsequent user studies in which 135 users interacted with the personalized and the original (non-personalized) version of the same Web environment.

\section{The PERsonaWeb PERSONALIZATION FrameWORK}

The PersonaWeb framework is composed of two main modules, the User Modeling and the Personalization modules.

\section{A. User Modeling Module}

The User Modeling module is primarily responsible to elicit the users' demographics (e.g., age, gender, etc.), and cognitive processing factors through a set of psychometric tools. The Verbal/Imager and Wholist/Analyst cognitive styles are elicited by exploiting two tests of Riding's CSA [8, 9], whereas the users' working memory capacity is extracted through a traditional visual working memory test (e.g., as utilized in [4, $6]$ ). In these tests, users are required to respond to a series of cognitive aptitude tasks (e.g., compare the similarity of a series of figures). Furthermore, the users' responses (accuracy and speed) are processed and accordingly, users are classified in a particular group, representing users that share the same cognitive processing characteristics.

Formalization and Data Processing. Let $U$ denote a set of users $\left\{u_{1}, u_{2}, \ldots, u_{n}\right\}$. Let $q_{j}^{c s}\left(u_{i}\right)$ denote a question $j$ that is part of a psychometric test for a specific cognitive stimulus $c S$ of the aforementioned tasks and is performed by user $u_{i}$. The result of $q_{j}^{c s}\left(u_{i}\right)$ is a quintuplet of the form $\left(c s, j, u_{i}, v a l, t\right)$, where $c s$ is the cognitive stimulus, $j$ is the question number, $u_{i}$ is the user, val is the answer (true or false) to the question and $t$ is the response time $(0<t \leq$ timeout $)$ for some predefined timeout period (e.g., 3 seconds). In this work, we consider the following cognitive stimulus $c s$ : verbal $(v)$, imager $(g)$, wholist $(w)$, analyst $(a)$, and visual working memory $(m a)$. Additionally, let the set of all correct questions (i.e., $v a l=t r u e$ ) for user $u_{i}$ for a specific cognitive stimulus $c s$ be denoted as $Q^{c s}\left(u_{i}\right)=\left\{q_{j}^{c s}\left(u_{i}\right): q . v a l=t r u e, \forall j\right\}$. We define the following:

- $\quad$ The average response time (rt) for a psychometric test of a specific cognitive stimulus $c s$ for user $u_{i}$ to be:

$$
r t^{c s}\left(u_{i}\right)=\frac{\sum_{\forall q_{j}^{c s}}\left(u_{i}\right) \in Q^{c s}\left(u_{i}\right)}{\left|Q^{c s}\left(u_{i}\right)\right|}
$$

- $\quad$ The average response time (RT) for a psychometric test of a specific cognitive stimulus $c s$ for all users to be:

$$
R T^{c s}=\frac{\sum_{\forall u_{i}} r t^{c s}\left(u_{i}\right)}{\left|\left\{u_{i}: r t^{c s}\left(u_{i}\right)>0\right\}\right|}
$$

- The number of correct responses (cr) of a specific cognitive stimulus $c s$ for user $u_{i}$ to be:

$$
\operatorname{cr}^{c s}\left(u_{i}\right)=\left|Q^{c s}\left(u_{i}\right)\right|
$$

We utilize the above definitions in order to infer two important ratios:
The Verbal/Imager Ratio $\lambda^{v: g}$ for user $u_{i}:$
$\lambda^{v: g}\left(u_{i}\right)=\left\{\begin{array}{lc}\frac{R T^{v}}{R T^{g}} & r t^{v}\left(u_{i}\right)=0, r t^{g}\left(u_{i}\right)=0 \\ \frac{R T^{v}}{r t^{g}\left(u_{i}\right)} & r t^{v}\left(u_{i}\right)=0 \\ \frac{r t^{v}\left(u_{i}\right)}{R T^{g}} & r t^{g}\left(u_{i}\right)=0 \\ \frac{r t^{v}\left(u_{i}\right)}{r t^{g}\left(u_{i}\right)} & \text { otherwise }\end{array}\right.$

- The Wholist/Analyst Ratio $\lambda^{w: a}$ for user $u_{i}$ :
$\lambda^{w: a}\left(u_{i}\right)=\left\{\begin{array}{lc}\frac{R T^{w}}{R T^{a}} & r t^{w}\left(u_{i}\right)=0, r t^{a}\left(u_{i}\right)=0 \\ \frac{R T^{w}}{r t^{a}\left(u_{i}\right)} & r t^{w}\left(u_{i}\right)=0 \\ \frac{r t^{w}\left(u_{i}\right)}{R T^{a}} & r t^{a}\left(u_{i}\right)=0 \\ \frac{r t^{w}\left(u_{i}\right)}{r t^{a}\left(u_{i}\right)} & \text { otherwise }\end{array}\right.$

The above ratios $\left(\lambda^{v: g}\left(u_{i}\right)\right.$ and $\left.\lambda^{w: a}\left(u_{i}\right)\right)$ indicate the users' cognitive style on the scales of Verbal-Imager and WholistAnalyst. Users with a low value of $\lambda^{v: g}\left(u_{i}\right)$ are considered to respond faster to the verbal types of stimuli, whereas users with a high value are considered to respond faster to the imager types of stimuli. Similarly, users with a low value of $\lambda^{w: a}\left(u_{i}\right)$ are considered to respond faster to the wholist types of stimuli, whereas users with a high value are considered to respond faster to the analyst types of stimuli. Finally, the total number of correct responses $\left(\mathrm{cr}^{\mathrm{ma}}\left(u_{i}\right)\right)$ of the visual working memory test indicates a user's working memory capacity with a low value indicating limited working memory capacity of that user, and a high value indicating an enhanced working memory capacity of that user. The ratios $\lambda^{v: g}\left(u_{i}\right), \lambda^{w: a}\left(u_{i}\right)$ and $c r^{m a}\left(u_{i}\right)$ for each user are then provided as input for cluster analysis, which is described in the next section, that aims to classify each user to a cognitive factor group.

User Classification. Cluster analysis is performed on the processed data with the aim to divide the set of users into cluster groups that are different from each other and whose members are similar to each other according to each of the cognitive factor values. We utilized the $k$-means clustering algorithm since it can yield very good results in our case as the data being processed are distinctively separated from each other depending on the responses to each cognitive stimulus of the users [4], as well as it is considered a robust and efficient clustering algorithm.

The $k$-means clustering results in constructing the final structure of the user model. Specifically, the user model um of a user $u_{i}\left(u m\left(u_{i}\right)\right)$ is composed of demographics (category $=d$ ) and cognitive characteristics (category $=c c$ ) and contains triplets of the form $(c t, c h, v a l)$, where $c t$ represents an information category (e.g., demographics, cognitive characteristics), ch represents a characteristic (e.g., age, gender, verbal/imager cognitive style, wholist/analyst cognitive style, working memory) and val the value of the specific characteristic. For example, a user $u_{i}$ may have the following user model: 


$$
\begin{gathered}
u m\left(u_{i}\right)=\{(d, \text { age }, 25),(c c, v / i, \text { verbal }), \\
(c c, w / a, \text { wholist }),(c c, w m, \text { limited })\}
\end{gathered}
$$

indicating that $u_{i}$ has an age $=25$ in the demographics $(d)$ information category and he/she is verbal $(v / i)$ and wholist $(w / a)$ with limited working memory $(\mathrm{wm})$ in the cognitive characteristics $(c c)$ category.

\section{B. Personalization Module}

Upon user classification, the Personalization module adapts semantically enriched content at run-time on the client's side. To accomplish this, the Personalization module utilizes: (i) the cognitive characteristics inside the constructed user model $u m\left(u_{i}\right)$ that was described in the previous section; (ii) semantically annotated Web content that can be provided in different formats; and (iii) adaptation rules that decide the "best-fit" design for a user $u_{i}$ according to the user model.

Content Management. The content management component, which is based on WordPress ${ }^{1}$, was developed to provide an easy to use tool for content providers to create semantically enriched Web-pages. Providers are able to annotate particular divisions of Web-pages indicating to the system which visible aspects of the E-Commerce environment should be adapted. The semantically enriched content (SEC) that is generated by this component can be roughly visualized as a set of triplets of the form $S E C=\left\{\left(p_{i}, s_{j}\right.\right.$, ctype $\left.)\right\}$, where $p_{i}$ is a unique page identifier, $s_{j}$ is a unique section identifier (i.e., a specific div tag) inside page $p_{i}$ and ctype is the content type (e.g., title, section) that can be adapted according to the rules incorporated in the adaptation engine that is described in the next section.

Adaptation Engine. The adaptation engine is composed of a number of adaptivity rules that can transform semantically enriched content according to the cognitive characteristics inside the user model $u m\left(u_{i}\right)$ of a user $u_{i}$. More specifically, the adaptation engine can be visualized as a function $a e\left(u m\left(u_{i}\right), p_{j}\right)$ where $u m\left(u_{i}\right)$ is the user model of a user $u_{i}$ and $p_{j}$ is a unique Web-page identifier. The adaptation engine starts by initializing an empty set of Web-page sections. Note that at the end of the procedure, this set will contain both adapted and non-adapted content that composes the specific Web-page. Next, the adaptation engine retrieves the content of all sections of the Web-page one by one. For each section, it checks if the specific section has semantically enriched content. If this is true, then it proceeds with adaptation of the content based on the cognitive characteristics that are stored in the user model of user $u_{i}$. In particular, several adaptation effects are applied on the semantically enriched content. If there is no semantically enriched content then the content is left unchanged. Finally, the adapted or non-adapted content for the specific section is communicated to the user interface.

To this end, the suggested designs and adaptation effects have been realized in an extended functional prototype of PersonaWeb. The interested reader may view all the available designs and adaptation effects through the PersonaWeb demo page $^{2}$.

\footnotetext{
${ }^{1}$ WordPress, http://www.wordpress.org

${ }^{2}$ PersonaWeb Demo Page, https://personaweb.cs.ucy.ac.cy/demo
}

\section{EXPERIMENTAL EVALUATION}

\section{A. Sampling and Procedure}

Two user studies were conducted in July 2014 and November 2014. A total of 135 participants (62 in Study \#1 and 73 in Study \#2) volunteered and consented to participate. Participants were recruited primarily at University laboratories, and they accessed a Web-based experimental E-Commerce environment which presented content based on an exact replica of the Hewlett Packard (HP) online shop ${ }^{3}$. Each session lasted approximately 45 minutes; where users spent 25 minutes on the user modeling process, and the remaining time to navigate in two different versions of the commercial Web environment (the original HP design (Figure 1) and the personalized design based on specific adaptation effects (Figure 2)).

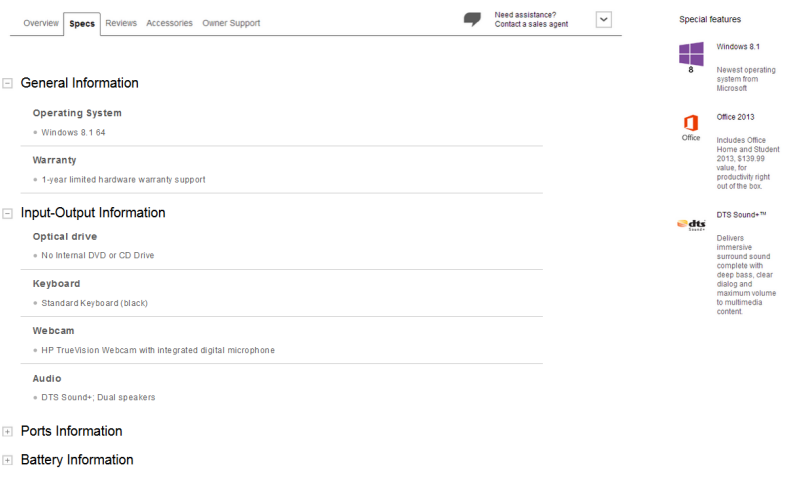

Figure 1. User interface of the original design.

Participants began the study by indicating their demographic information (e.g., age, gender, education level, online shopping experience) and cognitive characteristics via a set of specially designed psychometric tests. After completing the user modeling process, participants navigated in two different versions of the E-Commerce environment.

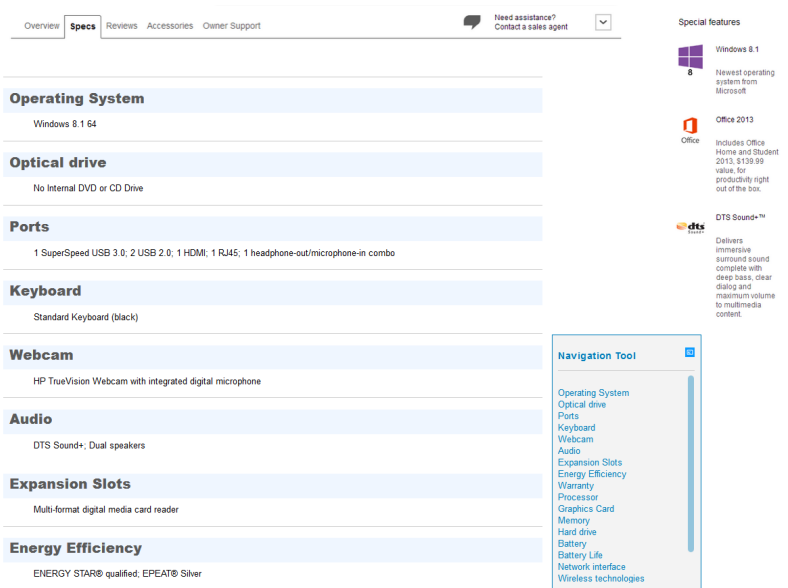

Figure 2. User interface of a personalized design (Imager-Analyst-limited working memory).

A within-subjects study design was followed; all users navigated sequentially in both versions of the same environment. The sequence of the two versions was randomly

${ }^{3}$ HP Online Shop, http://store.hp.com. Date extracted: March 2014 
provided to the participants with the aim to avoid familiarity or learnability effects. Based on a specific scenario, each user was required to navigate and answer three task-based questions in each design version (six in total) whose answers could be found in the Web content. The task-based questions were carefully designed to ensure the same level of complexity and understanding among them.

\section{B. Analysis of Results}

Based on the within-subjects study design, several paired samples $t$-test analyses were run to determine differences in terms of time to complete the task and success rate between user interactions with the original vs. the personalized design.

TABLE I. TASK EFFICIENCY AND EFFECTIVENESS OF STUDY \#1

\begin{tabular}{|l|c|c|c|c|c|c|}
\hline \multirow{2}{*}{ Outcome } & \multicolumn{2}{|c|}{ Original } & \multicolumn{2}{c|}{ Personalized } & \multicolumn{2}{|c|}{} \\
\cline { 2 - 7 } & M & SD & M & SD & n & t \\
\hline $\begin{array}{l}\text { Efficiency } \\
\text { (sec) }\end{array}$ & 56.19 & 58.38 & 32.82 & 30.50 & 62 & $3.38^{*}$ \\
\hline $\begin{array}{l}\text { Effectiveness } \\
(\%)\end{array}$ & 21.50 & 24.95 & 42.47 & 34.93 & 62 & $-4.67^{*}$ \\
\hline
\end{tabular}

a. $* \mathrm{p}<.01$

The results of Study $\# 1$ are displayed in Table 1 . Accordingly, there are statistically significant differences, at the .01 significance level, in original to personalized designs' scores for both task efficiency and effectiveness. More specifically, when users navigated in the original design, they completed the three tasks in a mean time of 56.19 seconds, in contrast to 32.82 seconds which were required to complete the tasks in the personalized design. In terms of effectiveness, results indicate that users had a task success rate of $21.50 \%$ in the original design as opposed to a $42.47 \%$ success rate in the personalized design. Table 2 presents the results of Study \#2, further indicating significant differences at the .01 (for task efficiency) and .05 (for task effectiveness) levels between original and personalized designs. Similarly, users navigating in the personalized design were able to complete the tasks much faster ( 58.70 seconds) as compared to the original design (106.57 seconds). In the same line, there was a noticeable difference in the task success rate between personalized and original designs ( $32.87 \%$ and $25.57 \%$ respectively).

TABLE II. TASK EFFICIENCY AND EFFECTIVENESS OF STUDY \#2

\begin{tabular}{|l|c|c|c|c|c|c|}
\hline \multirow{2}{*}{ Outcome } & \multicolumn{2}{|c|}{ Original } & \multicolumn{1}{c|}{ Personalized } & \multicolumn{1}{c|}{} \\
\cline { 2 - 7 } & M & SD & M & SD & n & t \\
\hline $\begin{array}{l}\text { Efficiency } \\
(\mathrm{sec})\end{array}$ & 106.57 & 79.39 & 58.70 & 41.69 & 73 & $5.62 * *$ \\
\hline $\begin{array}{l}\text { Effectiveness } \\
(\%)\end{array}$ & 25.57 & 23.90 & 32.87 & 29.12 & 73 & $-1.77 *$ \\
\hline
\end{tabular}

\section{CONCLUSIONS}

This paper presents an effort towards personalizing content and functionality of E-Commerce Web environments based on users' individual differences in cognitive processing. A personalization framework has been presented, placing emphasis on the user modeling and personalization modules, driven by specific cognitive processing factors of users for adapting the visual and interaction design aspects of ECommerce product views.
For the purpose of this research, two subsequent withinsubjects study designs were conducted in which 135 users in total interacted with the personalized and the original (nonpersonalized) version of the same Web environment with the aim to investigate the added value of personalizing content and functionality of E-Commerce product views in terms of task completion efficiency and effectiveness. The study findings provide initial evidence towards using the PersonaWeb framework for designing adaptive E-Commerce Web interfaces since task completion time and accuracy was significantly better in the personalized compared to the non-personalized version of the E-Commerce environment. Future research includes conducting user studies with larger samples and varying user profiles aiming to increase the external validity of this research. Furthermore, the proposed framework will be applied in different E-Commerce environments and interaction device types (desktop computers vs. touch-based) in order to cross-validate our findings and to increase the applicability of PersonaWeb in various contexts of use.

\section{ACKNOWLEDGMENT}

This work was partially carried out in the frame of the PersonaWeb project, funded by the Cyprus Research Promotion Foundation (ТПЕ/П НHРО/0311(BIE)/10), and the CogniWin project, funded by the EU Ambient Assisted Living Joint Program (AAL 2013-6-114).

\section{REFERENCES}

[1] A. Goy, L. Ardissono, and G. Petrone, "Personalization in e-commerce applications," in The Adaptive Web, 4321, P. Brusilovsky, A. Kobsa, and W. Nejdl, Eds. Heidelberg, Berlin, Springer-Verlag, 2007, pp. 485520.

[2] P. Brusilovsky, and E. Millán, "User models for adaptive hypermedia and adaptive educational systems," in The Adaptive Web, 4321, P. Brusilovsky, A. Kobsa, and W. Nejdl, Eds. Heidelberg, Berlin, SpringerVerlag, 2007, pp. 3-53.

[3] B. Steichen, M. Wu, D. Toker, C. Conati, and G. Carenini, "Te, te, hi, hi: Eye gaze sequence analysis for informing user-adaptive information visualizations," Proc. Springer Conference User Modeling, Adaptation, and Personalization (UMAP 14), Springer-Verlag, 2014, pp. 183-194.

[4] M. Belk, P. Germanakos, C. Fidas, and G. Samaras, "A personalisation method based on human factors for improving usability of user authentication tasks," Proc. Springer Conference User Modeling, Adaptation, and Personalization (UMAP 14), Springer-Verlag, 2014, pp. 13-24.

[5] S. Chen, and X. Liu, "An integrated approach for modeling learning patterns of students in web-based instruction: A cognitive style perspective," ACM Trans. J. Computer-Human Interaction, vol. 15, no. 1, article 1, 28 pages, 2008.

[6] P. Germanakos, N. Tsianos, Z. Lekkas, M. Belk, C. Mourlas, and G. Samaras, "Proposing web design enhancements based on specific cognitive factors: An empirical evaluation," Proc. IEEE Conference Web Intelligence and Intelligent Agent Technology (WI-IAT 09), IEEE Press, 2009, pp. 602-605.

[7] P. Germanakos, M. Belk, A. Constantinides, and G. Samaras, "The personaweb system: Personalizing e-commerce environments based on human factors," Proc. CEUR Workshops for the Conference User Modeling, Adaptation, and Personalization (UMAP 15), CEUR, 2015.

[8] R. Riding, "Cognitive styles analysis," Learning and Training Technology, Birmingham, UK, 1991.

[9] R. Riding, and I. Cheema, "Cognitive styles - An overview and integration," J. Educational Psychology, vol. 11, no. 3-4, pp. 193-215, 1991.

[10] A. Baddeley, "Working memory: Theories, models, and controversies," J. Annual Review Psychology, vol. 63, pp. 1-29, 2012. 